\title{
Microwave Assisted Biodiesel Production Using Heterogeneous Catalysts
}

\author{
Haris Mahmood Khan ${ }^{1, *}$, Tanveer Iqbal ${ }^{1}$, M. A. Mujtaba ${ }^{2} \mathbb{D}$, Manzoore Elahi M. Soudagar ${ }^{3} \mathbb{D}, I_{b h a m ~} \operatorname{Veza}^{4}(\mathbb{D}$ \\ and I. M. Rizwanul Fattah $5, *$ (i) \\ 1 Department of Chemical, Polymer and Composite Materials Engineering, \\ University of Engineering and Technology (New Campus), Lahore 54890, Pakistan; tanveer@uet.edu.pk \\ 2 Department of Mechanical Engineering, University of Engineering and Technology (New Campus), \\ Lahore 54890, Pakistan; m.mujtaba@uet.edu.pk \\ 3 Department of Mechanical Engineering, School of Technology, Glocal University, Delhi-Yamunotri Marg, \\ SH-57, Mirzapur Pole, Saharanpur 247121, India; me.soudagar@gmail.com \\ 4 Automotive Development Centre, School of Mechanical Engineering, Universiti Teknologi Malaysia, \\ Johor Bahru 81310, Malaysia; ibhamv@gmail.com \\ 5 Centre for Green Technology, Faculty of Engineering and IT, University of Technology Sydney, \\ Ultimo, NSW 2007, Australia \\ * Correspondence: hariskhan@uet.edu.pk (H.M.K.); IslamMdRizwanul.Fattah@uts.edu.au (I.M.R.F)
}

check for updates

Citation: Khan, H.M.; Iqbal, T.; Mujtaba, M.A.; Soudagar, M.E.M.; Veza, I.; Fattah, I.M.R. Microwave Assisted Biodiesel Production Using Heterogeneous Catalysts. Energies 2021, 14, 8135. https://doi.org/ $10.3390 /$ en14238135

Academic Editor: Diego Luna

Received: 2 November 2021

Accepted: 29 November 2021

Published: 4 December 2021

Publisher's Note: MDPI stays neutral with regard to jurisdictional claims in published maps and institutional affiliations.

Copyright: (c) 2021 by the authors. Licensee MDPI, Basel, Switzerland. This article is an open access article distributed under the terms and conditions of the Creative Commons Attribution (CC BY) license (https:// creativecommons.org/licenses/by/ $4.0 /)$.

\begin{abstract}
As a promising renewable fuel, biodiesel has gained worldwide attention to replace fossil-derived mineral diesel due to the threats concerning the depletion of fossil reserves and ecological constraints. Biodiesel production via transesterification involves using homogeneous, heterogeneous and enzymatic catalysts to speed up the reaction. The usage of heterogeneous catalysts over homogeneous catalysts are considered more advantageous and cost-effective. Therefore, several heterogeneous catalysts have been developed from variable sources to make the overall production process economical. After achieving optimum performance of these catalysts and chemical processes, the research has been directed in other perspectives, such as the application of non-conventional methods such as microwave, ultrasonic, plasma heating etc, aiming to enhance the efficiency of the overall process. This mini review is targeted to focus on the research carried out up to this date on microwave-supported heterogeneously catalysed biodiesel production. It discusses the phenomenon of microwave heating, synthesis techniques for heterogeneous catalysts, microwave mediated transesterification reaction using solid catalysts, special thermal effects of microwaves and parametric optimisation under microwave heating. The review shows that using microwave technology on the heterogeneously catalysed transesterification process greatly decreases reaction times (5-60 $\mathrm{min}$ ) while maintaining or improving catalytic activity (>90\%) when compared to traditional heating.
\end{abstract}

Keywords: microwave-assisted transesterification; heterogeneous catalyst; biodiesel; reaction time; thermal effects

\section{Introduction}

In the twenty-first century, it has become a significant constraint for the scientific community to rigorously address the present energy issue while also taking into account ecological concerns about global climate change. The threat of diminishing fossil fuels and ongoing global warming challenges prompted scientists to search for sustainable and renewable energy resources to address the energy demands of the world's growing population. As a result, eco-friendly and renewable fuels are recommended to be sought after and investigated at this time. Biofuels such as biodiesel and bioethanol are proposed as renewable fuels obtained from renewable resources that may be used to replace fossilderived diesel and gasoline fuels [1-4]. In addition to ensuring the availability of renewable 
and sustainable fuels, their quick production techniques must be discovered to fulfil the demands of the rising population.

Transesterification is the most prominent method generally used for biodiesel generation $[1,5]$. It entails the deployment of homogeneous, heterogeneous or enzymatic catalysts to speed up the reaction [6,7]. Although homogeneous catalysts demonstrate good catalytic activity in transesterification, the difficulties associated with their removal from the mixture and additional washing stages implied extra operational expenses and hence made the overall process less cost-effective [8,9]. These issues can be effectively resolved by the application of heterogeneous catalysts $[10,11]$. A number of heterogeneous catalysts, either synthetic or derived from waste resources, have been developed to effectively transform oil sources into biodiesel [12-16]. After achieving the optimal performance of these catalysts, research has now shifted in other directions, such as applying non-conventional techniques such as plasma, ultrasonic, microwave-assisted methods for getting rapid conversions [11].

The electromagnetic rays with wavelengths of $0.01-1 \mathrm{~m}$ along with frequency varying from 0.3 to $300 \mathrm{GHz}$ are called microwave irradiation. For instance, radar transmissions operate at wavelengths ranging from 0.01 to $0.25 \mathrm{~m}$ and telecommunications practice the spare portion of wavelengths. All household microwave ovens and reactors for chemical manufacturing function at a frequency of $2.45 \mathrm{GHz}$, holding a wavelength of $12.25 \mathrm{~cm}$ with attention to evade the interferences with mobile phone frequencies and telecommunications. The microwave region stays between infrared and radio waves [17]. The electromagnetic spectrum is seen in Figure 1. At the right, the tiny visible light range is expanded.

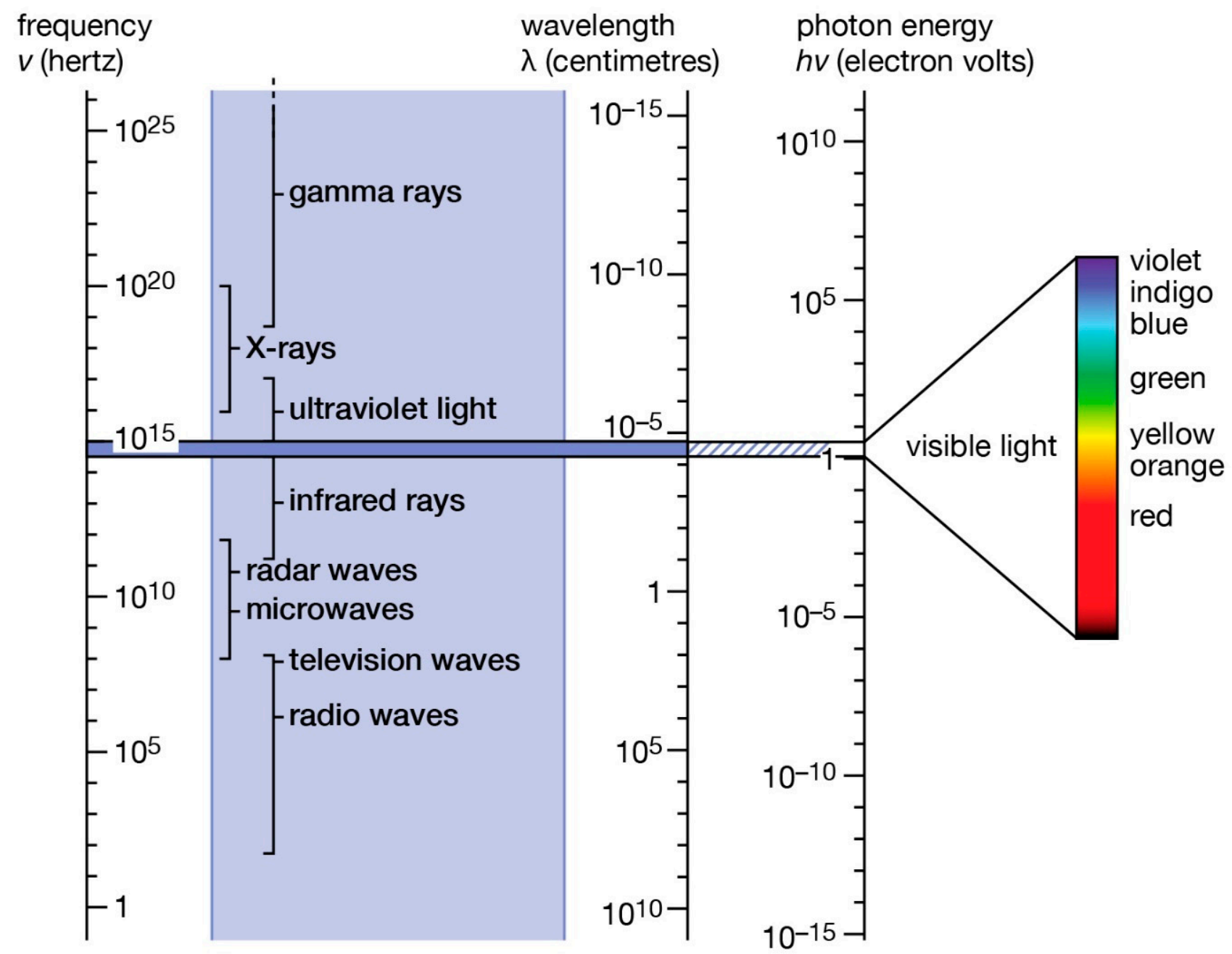

Figure 1. Electromagnetic spectrum and their corresponding wavelengths and frequencies. 
Microwaves improve and accelerate chemical reactions because of the direct accessibility of energy to the reactants. In the case of microwaves, heat transfer is even more efficient than normal heating, therefore leading to short reaction times [18]. In the case of normal heating, an oil bath or furnace is employed, which initially heats the walls of the container by conduction or convection and later heat is transmitted to the centre of the sample. At the same time, microwaves heat the target compounds without heating oil bath or furnace, which conserves energy and time. It boosts the reaction speed and makes the separation mechanism simpler in comparison with normal heating. It has been stated that the reaction accelerates via thermal effect only, also called the kinetic effect, which is associated with instant temperature rise during the ionic conduction/dipolar polarisation under microwave heating $[19,20]$. It has also been concluded that microwave heating also significantly lessened the reaction time while keeping the kinetic mechanisms of the same reactions alike. The other advantages of microwave heating include selective heating, diffusional radiation, reduced adverse emissions, clean energy and reaction trend, molecular level heating, etc. [21]. In addition, along with the utilisation of efficient technologies, optimisation of reaction parameters is still necessary to get the highest yield of biodiesel. Nowadays, various researchers have also targeted the application of different techniques such as artificial neural network (ANN), adaptive neuro-fuzzy inference system (ANFIS) and response surface methodology (RSM) and hybrid approaches such as support vector machine-RSM (SVM-RSM) and extreme learning machine-RSM (ELM-RSM) to optimise reaction parameters to get the maximum yields of biodiesel [22,23].

This mini review has concentrated on reviewing the research carried out to date on microwave-assisted heterogeneously catalysed biodiesel generation. It incorporates the phenomenon of microwave heating, synthesis techniques for heterogeneous catalysts, microwave mediated transesterification reaction using solid catalysts, special thermal effects of microwaves and parametric optimisation under microwave heating. It suggests that the application of microwave technology on the heterogeneously catalysed transesterification process significantly reduces the reaction times with better catalytic activity compared to conventional heating.

\section{Synthesis Techniques for Heterogeneous Catalysts}

There are a variety of techniques that have been employed in the literature for the manufacturing of heterogeneous catalysts ranging from the direct use of material after grinding to the other modification techniques such as calcination, physical mixing, bifunctional modification, wet impregnation, co-precipitation, calcination-hydration-dehydration, and sol-gel method etc. [24]. Either heterogeneous catalysts can be produced synthetically from chemicals or can be derived from waste resources such as eggshells, waste bones and other agricultural waste. For instance, some of the heterogeneous catalysts based on metal and mixed metal oxides such as calcium oxide $(\mathrm{CaO})$, strontium oxide $(\mathrm{SrO})$, magnesium oxide (MgO), calcium-diglyceroxide, calcium methoxide, aluminium oxidesbased catalysts and sulphated zirconia, etc., are commercially available and others such as $\mathrm{LiAlO}_{2}, \mathrm{Li}_{4} \mathrm{SiO}_{4}$ etc., can be synthesised from variable sources by adopting suitable techniques for catalysts synthesis [25-29]. Moreover, nowadays, it has become decisive to carefully select sustainable and renewable sources from which heterogeneous catalysts should be prepared to avoid the ill effects on the environment. Therefore, research has been directed towards waste-based resources and environmentally friendly techniques for catalyst synthesis. The synthesis methods for heterogeneous catalysts, along with their brief description, are provided in Table 1. 
Table 1. Categorisation of heterogeneous catalysts synthesis methods.

\begin{tabular}{|c|c|c|}
\hline Method & Description & Ref. \\
\hline Impregnation method & $\begin{array}{l}\text { A solution of precursor/active components is } \\
\text { taken, and carrier is immersed in it. After a } \\
\text { while, when equilibrium is achieved, the } \\
\text { remaining liquid is withdrawn and the catalyst } \\
\text { is acquired following the drying, calcination and } \\
\text { activation }\end{array}$ & [30] \\
\hline Precipitation & $\begin{array}{l}\text { A precipitation agent is added in an aqueous } \\
\text { solution of metallic salts to obtain the crystal of } \\
\text { carbonates, hydrated oxides, or gels. }\end{array}$ & [31] \\
\hline $\begin{array}{l}\text { Precipitation-impregnation } \\
\text { method }\end{array}$ & $\begin{array}{l}\text { A method based on the combination of the } \\
\text { impregnation and precipitation technique } \\
\text { initially synthesised the precipitant matrix in the } \\
\text { impregnation solution. Upon completion of } \\
\text { impregnation, the precipitant is accumulated on } \\
\text { the carrier's surface through heating. }\end{array}$ & [32] \\
\hline Chemical deposition method & $\begin{array}{l}\text { The films are produced on the substrate surface } \\
\text { via chemical reactions using materials containing } \\
\text { film components. }\end{array}$ & [33] \\
\hline Sol-gel method & $\begin{array}{l}\text { The butyl titanate and anhydrous ethanol are } \\
\text { mixed slowly to another solution of } \\
\text { demineralised water, anhydrous ethanol, nitric } \\
\text { acid and suitable quantity of nitrate precursor at } \\
\text { ambient temperature under strong mixing to } \\
\text { perform hydrolysis }\end{array}$ & [34] \\
\hline Physical mixing & $\begin{array}{l}\text { The multiple materials in a ground form are well } \\
\text { mixed or blended }\end{array}$ & [34] \\
\hline Calcination & $\begin{array}{l}\text { The substance is exposed to elevated } \\
\text { temperature in a furnace }\end{array}$ & [34] \\
\hline
\end{tabular}

\section{Microwave Assisted Transesterification Using Heterogeneous Catalysts}

The transesterification reaction involves oil, alcohol and catalyst, which are vigorously mixed under a specified temperature for the reaction to proceed. In the case of microwave aided biodiesel production, these three components are mixed and processed under microwave irradiation [35]. Upon completion of the reaction, biodiesel is separated and purified. Methanol is preferably used for microwave-assisted reactions because of its high polarity and capability to absorb microwaves. The transesterification procedure may be enhanced through ionic conduction and dipolar polarisation under microwave heating, which may have a higher reliance on the absorption ability and polarity of the reacting species [20]. Heterogeneously catalysed transesterification under microwave irradiation displayed short reaction times with better biodiesel yields as displayed in Table 2 .

For instance, catalysts derived from waste eggshells provided more than 95\% yield of biodiesel within 4 min under microwave mediated transesterification reaction [36]. In another study, corn cobs derived sulfonated activated carbon was utilised as a solid catalyst for microwave supported reaction process of soybean oil. The FAME yield of $88.7 \%$ was attained in $20 \mathrm{~min}$ with methanol/oil ratio of 6:1. It indicates the effectiveness of the heterogeneously catalysed transesterification reaction in the occurrence of microwaves [37]. Microwave supported transesterification of yellow horn oil utilising heteropolyacid catalyst depicted more than $96 \%$ yield of biodiesel in $10 \mathrm{~min}$ with catalyst amount of only $1 \mathrm{wt} . \%$ [38]. Similarly, in another investigation, Guinea fowl bone-based heterogeneous catalyst displayed $95.82 \%$ biodiesel yield within $20 \mathrm{~min}$ in the transesterification of oil (Annona squamosa L. seed) using microwaves [39]. In another place, natural hydroxyapatite synthesised from pork bone was applied as solid catalyst in microwave supported 
transesterification of jatropha oil [40]. The greatest yield of $94 \%$ was achieved in $5 \mathrm{~min}$ using microwave power of $800 \mathrm{~W}\left(\mathrm{CH}_{3} \mathrm{OH} /\right.$ oil: $18 / 1$, catalyst weight: $\left.4 \%\right)$. Additionally, the investigations mentioned above suggest a significant reduction in reaction times in the presence of microwaves while using heterogeneously catalysed systems for biodiesel generation.

Hsiao et al. [41] analysed the microwave mediated transesterification of soybean oil into methyl esters depicting above $95 \%$ yield of biodiesel while employing nano-powder $\mathrm{CaO}$ as a heterogeneous catalyst. As per their studies, microwave heating was appeared to be more proficient than a traditional bath for biodiesel generation. Patil et al. [42] studied the reaction kinetics of transesterification of oil (Camelina sativa) using metal oxides catalysts such as oxides of Barium, calcium, magnesium and strontium under the microwave and conventional heating conditions. The comparison between both heating methods indicated that the $\mathrm{k}$ values for the microwave-mediated process were of two orders of magnitude higher in comparison with the traditional heating scheme. It has been established that the heating technique displays a substantial effect in bettering the reaction kinetics.

In another study, Gupta and his coworkers [43] attempted to adjust the reaction parameters in the microwave mediated transesterification of WCO involved biodiesel generation using calcium diglyceride as a heterogeneous catalyst. As per their studies, under the optimised reaction variables such as methanol/oil 7.46:1, catalyst weightage of $1.03 \%$, the temperature of $62{ }^{\circ} \mathrm{C}$, the highest biodiesel yield of $94.86 \%$ was attained within 15 min using microwave heating whereas only $42.59 \%$ yield was achieved under the same reaction parameters using normal heating process.

The transesterification of palm oil employing hydrated heat-treated seashells as a heterogeneous catalyst has been examined for microwave heating and conventional heating conditions [44]. It was detected that yield of only 59.2\% was achieved after $2 \mathrm{~h}$ in the conventional reactor at high temperature and pressure, whereas under microwave heating, the performance of the same catalyst enhanced, and a maximum yield of $96 \%$ was obtained after $10 \mathrm{~min}$ with a catalyst weight of only $0.5 \mathrm{~g}$.

Therefore, it can be deduced from the studies that solid catalysts mediated reaction process occurring under microwaves displays short reaction times and better yields as compared to normal heating while using the same catalysts. Alongside, the microwave heating mechanism improves reaction kinetics by increasing rate constant values, which results in better conversions.

Table 2. Synopsis of recent works reported for microwave mediated biodiesel generation using various heterogeneous catalysts.

\begin{tabular}{|c|c|c|c|c|c|c|}
\hline Materials & Catalyst Preparation & Catalyst & Oil Used & $\begin{array}{c}\text { Reaction } \\
\text { Parameters }\end{array}$ & Yield & Ref. \\
\hline Waste eggshells & $\begin{array}{l}\text { Calcined at } 800{ }^{\circ} \mathrm{C} \text { for } 4 \mathrm{~h} \text { in } \\
\text { air atmosphere }\left(10^{\circ} \mathrm{C} / \mathrm{min}\right) \text {. }\end{array}$ & $\mathrm{CaO}$ & Palm olein oil & $\begin{array}{c}\text { M/O: } 18, \\
\text { catalyst: } \\
15 \text { wt. } \% \text {, time: } \\
4 \text { min, } \\
\text { temperature: -, } \\
\text { mic. power: } \\
900 \mathrm{~W}\end{array}$ & $Y=96.7$ & [36] \\
\hline Corn cobs & $\begin{array}{c}\text { First, activated carbon was } \\
\text { obtained from corn cobs and } \\
\text { then sulfonated by applying } \\
\text { sulphonating agent. }\end{array}$ & $\begin{array}{l}\text { Sulfonated } \\
\text { activated } \\
\text { carbon }\end{array}$ & Soybean oil & $\begin{array}{c}\text { M/O: 6, } \\
\text { catalyst: } \\
20 \text { wt.\%, time: } \\
20 \text { min, } \\
\text { temperature: -, } \\
\text { mic. power: } \\
\text { 0-600 W }\end{array}$ & $\mathrm{Y}=88.7$ & [37] \\
\hline
\end{tabular}


Table 2. Cont.

\begin{tabular}{|c|c|c|c|c|c|c|}
\hline Materials & Catalyst Preparation & Catalyst & Oil Used & $\begin{array}{l}\text { Reaction } \\
\text { Parameters }\end{array}$ & Yield & Ref. \\
\hline $\begin{array}{l}\text { Waste shells of } \\
\text { oyster and } \\
\text { Pyramidella }\end{array}$ & $\begin{array}{l}\text { Calcined at } 900{ }^{\circ} \mathrm{C} \text { for } 2 \mathrm{~h} \text { in } \\
\text { air environment }\left(10^{\circ} \mathrm{C} / \mathrm{min}\right)\end{array}$ & $\mathrm{CaO}$ & $\begin{array}{c}\text { Jatropha curcas } \\
\text { oil }\end{array}$ & $\begin{array}{c}\text { M/O: } 15, \\
\text { catalyst: } 4 \mathrm{wt} \% \text {, } \\
\text { time: } 5 \mathrm{~min}, \\
\text { temperature: -, } \\
\text { mic. power: } \\
800 \mathrm{~W}\end{array}$ & $Y=93$ & [45] \\
\hline $\begin{array}{c}\mathrm{ZrO}_{2^{-}} \\
\text {supported } \\
\text { bamboo leaf } \\
\text { ash }\end{array}$ & $\begin{array}{l}\text { First, addition of } \mathrm{ZrOCl} \\
\text { solution to isopropanol } \\
\text { solvent and water while } \\
\text { stirring to get the precursor } \\
\mathrm{ZrO}_{2} \text { solution. Afterward, the } \\
\text { mixing of the suspension of } \\
\text { precursor solution and leaf ash } \\
\text { accompanied by adding } \mathrm{HCl} \\
\text { and then refluxing for } 4 \mathrm{~h} \text { is } \\
\text { needed to get } \mathrm{ZrO}_{2} / \text { ash } \\
\text { composite. The mixture was } \\
\text { then dried and calcined. }\end{array}$ & $\begin{array}{c}\text { Silica-based } \\
\text { material with } \\
\text { acid properties } \\
\text { of } \mathrm{ZrO}_{2}\end{array}$ & Soybean oil & $\begin{array}{c}\text { M/O: } 15, \\
\text { catalyst:12 } \\
\text { wt.\%, time: } \\
30 \text { min, } \\
\text { temperature: -, } \\
\text { mic. assisted }\end{array}$ & $Y>90$ & [46] \\
\hline $\begin{array}{l}\text { Cassava peel } \\
\text { derived } \\
\text { sulfonated acid } \\
\text { catalyst }\end{array}$ & $\begin{array}{l}\text { Initially, the biomass pyrolysis } \\
\text { (slow) was carried out at } \\
40{ }^{\circ} \mathrm{C} \text { for } 1 \mathrm{~h} \text {. After that, the } \\
\text { sulfonation for the catalyst } \\
\text { functionalisation was executed } \\
\text { by introducing } \mathrm{SO}_{3} \mathrm{H} \text { groups } \\
\text { into biochar to get } \\
\text { biochar-based acid catalyst. } \\
\text { The co-precipitation technique } \\
\text { was applied for the synthesis } \\
\text { of Fe } \mathrm{O}_{4} \text { magnetic } \\
\text { nanoparticles. The } \\
\text { nanomagnetic biocatalyst was } \\
\text { synthesised by mixing } \\
\text { magnetic nanoparticles and } \\
\text { sulfonated biochar catalyst in } \\
\text { demineralised } \mathrm{H}_{2} \mathrm{O} \text { utilising } \\
\text { an ultrasonicator at an } \\
\text { ambient temperature for } 8 \mathrm{~h} \text {. } \\
\text { Later, the nanomagnetic } \\
\text { biocomposite was dried and } \\
\text { stored in a desiccator. }\end{array}$ & $\begin{array}{l}\text { weak hydroxyl } \\
\text { and strong } \\
\text { carboxylic and } \\
\text { sulfonic acidic } \\
\text { groups }\end{array}$ & $\begin{array}{c}\text { Millettia } \\
\text { pinnata seed oil }\end{array}$ & $\begin{array}{l}\text { M/O: } 11, \\
\text { catalyst: } 3 \text { wt. } \% \text {, } \\
\text { time: } 45 \text { min, } \\
\text { temperature: -, } \\
\text { mic. assisted }\end{array}$ & $\mathrm{Y}=98.7$ & [47] \\
\hline $\begin{array}{l}\text { Heteropolyacid } \\
\text { catalyst }\end{array}$ & $\begin{array}{l}\text { The catalyst was purchased as } \\
\text { it is. }\end{array}$ & $\mathrm{Cs}_{2.5} \mathrm{H}_{0.5} \mathrm{PW}_{12} \mathrm{O}_{40}$ & Yellow horn oil & $\begin{array}{l}\text { M/O: } 12, \\
\text { catalyst: } 1 \mathrm{wt} . \%, \\
\text { time: } 10 \mathrm{~min}, \\
\text { temperature: -, } \\
\text { mic. power: } \\
500 \mathrm{~W}\end{array}$ & $Y=96.22$ & [38] \\
\hline $\begin{array}{c}\text { Potassium } \\
\text { fluoride }(\mathrm{KF}) \\
\text { modification of } \\
\text { hydrotalcite } \\
\left(\text { Sorbacite }^{\circledR}\right)\end{array}$ & $\begin{array}{l}\text { The solid reaction technique } \\
\text { was used for the catalyst } \\
\text { production. The mixture of } \\
\text { potassium fluoride and } \\
\text { hydrotalcite was milled and } \\
\text { heated at a specified } \\
\text { temperature for } 2 \mathrm{~h} . \mathrm{KF} \\
\text { content was differed from } \\
5-30 \mathrm{wt} \% \text { in range. }\end{array}$ & $\begin{array}{l}\text { KF modified } \\
\text { hydrotalcite }\end{array}$ & Jatropha oil & $\begin{array}{c}\text { M/O: } 2-10, \\
\text { catalyst: } 3.33,5, \\
10 \text { wt.\%, time: } \\
\text { 2-30 min, } \\
\text { temperature: -, } \\
\text { mic. power: } \\
\text { 0-900 W }\end{array}$ & $Y=97$ & [48] \\
\hline
\end{tabular}


Table 2. Cont.

\begin{tabular}{|c|c|c|c|c|c|c|}
\hline Materials & Catalyst Preparation & Catalyst & Oil Used & $\begin{array}{c}\text { Reaction } \\
\text { Parameters }\end{array}$ & Yield & Ref. \\
\hline $\begin{array}{c}\mathrm{KOH} \\
\text { impregnated } \\
\text { CaO catalyst }\end{array}$ & $\begin{array}{l}\mathrm{KOH} \text { impregnated calcium } \\
\text { oxide }(\mathrm{CaO}) \text { catalysts were } \\
\text { synthesised by wet } \\
\text { impregnation method }\end{array}$ & $\begin{array}{l}20 \% \mathrm{KOH} \\
\text { impregnation } \\
\text { on } \mathrm{CaO}\end{array}$ & $\begin{array}{c}\text { Jatropha curcas } \\
\text { oil }\end{array}$ & $\begin{array}{c}\text { M/O: } 8.42, \\
\text { catalyst: } \\
3.17 \text { wt. } \% \text {, time: } \\
67.9 \text { min, } \\
\text { temperature: -, } \\
\text { mic. assisted }\end{array}$ & $\mathrm{Y}=97.1$ & [49] \\
\hline $\begin{array}{l}\text { Guinea fowl } \\
\text { bone }\end{array}$ & Calcined at $900^{\circ} \mathrm{C}$ for $5 \mathrm{~h}$ & $\beta-\mathrm{Ca}_{3}\left(\mathrm{PO}_{4}\right)_{2}$ & $\begin{array}{l}\text { Annona } \\
\text { squamosa L. } \\
\text { seed oil }\end{array}$ & $\begin{array}{c}\text { M/O: } 18, \\
\text { catalyst: } 4 \text { wt. } \% \text {, } \\
\text { time: } 20 \text { min, } \\
\text { temperature: -, } \\
\text { mic. power: } \\
800 \mathrm{~W}\end{array}$ & $\mathrm{Y}=95.82$ & [39] \\
\hline $\begin{array}{l}\text { Lignin based } \\
\text { heterogeneous } \\
\text { solid acid } \\
\text { catalyst } \\
\text { extracted from } \\
\text { sugarcane } \\
\text { bagasse }\end{array}$ & $\begin{array}{l}\text { The alkaline pulping method } \\
\text { was employed for lignin } \\
\text { extraction from bagasse } \\
\text { followed by the partial } \\
\text { carbonisation in a furnace at } \\
400{ }^{\circ} \mathrm{C} \text {. Later, the product was } \\
\text { mixed with conc. } \mathrm{H}_{2} \mathrm{SO}_{4} \text { at a } \\
\text { specified temperature for } 2 \mathrm{~h} \text {. }\end{array}$ & $\begin{array}{l}\text { Sulfonic group, } \\
\text { carboxyl group } \\
\text { and hydroxyl } \\
\text { group after } \\
\text { effective } \\
\text { chemical } \\
\text { activation and } \\
\text { sulfonation. }\end{array}$ & $\begin{array}{l}\text { Waste cooking } \\
\text { oil }\end{array}$ & $\begin{array}{c}\text { M/O: } 18, \\
\text { catalyst: } \\
15 \text { wt. } \% \text {, time: } \\
15 \text { min, } \\
\text { temperature: -, } \\
\text { mic. assisted }\end{array}$ & $\mathrm{Y}=89.19$ & [50] \\
\hline $\begin{array}{l}\text { Alumina/silica } \\
\text { loaded with } \\
\text { potassium } \\
\text { sodium tartrate }\end{array}$ & $\begin{array}{l}\text { A one-step sol-gel method was } \\
\text { employed for catalyst } \\
\text { preparation. In the end, the } \\
\text { catalyst was heat-treated at } \\
\text { elevated temperature for } 5 \mathrm{~h} \text {. }\end{array}$ & $\begin{array}{c}\text { Good } \\
\text { dispersion of } \\
\mathrm{C}_{4} \mathrm{H}_{4} \mathrm{O}_{6} \mathrm{KNa} \text { on } \\
\text { the support }\end{array}$ & Soybean oil & $\begin{array}{c}\text { M/O: } 13, \\
\text { catalyst: } 8 \text { wt. } \% \text {, } \\
\text { time: } 45 \text { min, } \\
\text { temperature: } \\
65^{\circ} \mathrm{C} \text {, mic. } \\
\text { assisted }\end{array}$ & $Y=96.5$ & [51] \\
\hline $\begin{array}{l}\text { A sulfonated- } \\
\text { glucose solid } \\
\text { acid catalyst }\end{array}$ & $\begin{array}{l}\text { The incomplete carbonisation } \\
\text { of a specific quantity of } \\
\text { D-glucose was carried out } \\
\text { while heating at } 400{ }^{\circ} \mathrm{C} \text { for } \\
12 \mathrm{~h} \text { under } \mathrm{N}_{2} \text { gas. The } \\
\text { product was ground and } \\
\text { heated at } 160{ }^{\circ} \mathrm{C} \text { under inert } \\
\text { atmosphere for } 12 \mathrm{~h} \text { in the } \\
\text { presence of conc. } \mathrm{H}_{2} \mathrm{SO}_{4} \text {. } \\
\text { Lastly, the suspension was } \\
\text { further diluted with distilled } \\
\text { water and the precipitate was } \\
\text { acquired. }\end{array}$ & $\begin{array}{l}\text { Glucose- } \mathrm{SO}_{3} \mathrm{H} \\
\text { catalyst }\end{array}$ & $\begin{array}{l}\text { Non-edible } \\
\text { palm fatty acid } \\
\text { distillate }\end{array}$ & $\begin{array}{c}\text { M/O: } 12, \\
\text { catalyst: } 3 \text { wt. } \%, \\
\text { time: } 15 \text { min, } \\
\text { temperature: } \\
75^{\circ} \mathrm{C}, \text { mic. } \\
\text { assisted }\end{array}$ & $Y=96$ & [52] \\
\hline $\begin{array}{l}\text { Potassium } \\
\text { hydroxide } \\
(\mathrm{KOH}) \\
\text { impregnated } \\
\text { alumina } \\
\text { catalysts }\end{array}$ & $\begin{array}{l}\text { Wet impregnation method was } \\
\text { employed for } \mathrm{KOH} / \gamma-\mathrm{Al}_{2} \mathrm{O}_{3} \\
\text { catalysts preparation using the } \\
\gamma-\mathrm{Al}_{2} \mathrm{O}_{3} \text { support. The } \\
\text { powdery material was } \\
\text { heat-treated in a tube furnace } \\
\text { under the air environment at } \\
\text { the specified time and } \\
\text { temperature. }\end{array}$ & $\mathrm{K}_{2} \mathrm{O}$ & Soybean oil & $\begin{array}{c}\text { M/O: } 12, \\
\text { catalyst: } 3 \text { wt. } \% \text {, } \\
\text { time: } 35 \text { min, } \\
\text { temperature: } \\
65^{\circ} \mathrm{C} \text {, mic. } \\
\text { assisted }\end{array}$ & $\mathrm{Y}>95$ & [53] \\
\hline $\begin{array}{l}\text { Ionic liquid } \\
\text { (IL)-microwave } \\
\text { heating }\end{array}$ & - & - & $\begin{array}{c}\text { Wet } \\
\text { Nannochloropsis } \\
\text { sp. biomass }\end{array}$ & $\begin{array}{c}\text { M/O: } 4, \\
\text { catalyst: -, time: } \\
14 \text { min, } \\
\text { temperature: -, } \\
\text { mic. assisted, IL } \\
\text { ratio } \\
\text { maintained at } \\
\text { 1:0.5 }\end{array}$ & $Y=42.22$ & [54] \\
\hline
\end{tabular}


Table 2. Cont.

\begin{tabular}{|c|c|c|c|c|c|c|}
\hline Materials & Catalyst Preparation & Catalyst & Oil Used & $\begin{array}{c}\text { Reaction } \\
\text { Parameters }\end{array}$ & Yield & Ref. \\
\hline $\begin{array}{l}\text { Strontium } \\
\text { Oxide } \\
\text { Agglomerates } \\
\text { Depositing } \\
\text { upon Titanium } \\
\quad \text { Plate }\end{array}$ & $\begin{array}{l}\text { Strontium oxide powder } \\
\text { deposited on a titanium plate } \\
\text { picked as the support because } \\
\text { of the possible formation of } \\
\mathrm{SrTiO}_{3} \text { at their interface after } \\
\text { heat treatment }\end{array}$ & $\mathrm{SrO} / \mathrm{TiO}_{2} \_\mathrm{P}$ & Olive oil & $\begin{array}{l}\text { M/O: } 6 \text {, } \\
\text { catalyst: } 3 \text { wt. } \%, \\
\text { time: } 4 \text { min, } \\
\text { temperature: -, } \\
\text { mic. assisted }\end{array}$ & $C=87.7$ & [55] \\
\hline $\begin{array}{l}\text { Metal Oxide } \\
\text { catalysts }\end{array}$ & Barium oxide & $\mathrm{BaO}$ & $\begin{array}{c}\text { Camelina sativa } \\
\text { oil }\end{array}$ & $\begin{array}{c}\text { M/O: } 9, \\
\text { catalyst: } \\
1.5 \text { wt.\%, time: } \\
4 \text { min, } \\
\text { temperature: -, } \\
\text { mic. assisted }\end{array}$ & $Y=94$ & [42] \\
\hline $\begin{array}{c}\text { The } \\
\text { propyl-SO } \\
\text { amorphous } \\
\mathrm{SiO}_{2}\end{array}$ & $\begin{array}{l}\text { The sol-gel technique was } \\
\text { employed for the preparation } \\
\text { of the propyl-SO }{ }_{3} \mathrm{H} \\
\text { (10 wt.\%)/ silica. The specified } \\
\text { quantity of tetraethyl } \\
\text { orthosilicate was added in } \\
\text { ethanol and kept for stirring for } \\
15 \text { min at } 45^{\circ} \mathrm{C} \text {. Next, a specific } \\
\text { quantity of acetic acid aqueous } \\
\text { solution (pH:5) was put on the } \\
\text { mixture, followed by } \\
\text { 3-mercaptopropyltrimethoxy } \\
\text { silane and hydrogen peroxide. } \\
\text { The solution was continuously } \\
\text { mixed at } 45^{\circ} \mathrm{C} \text { overnight under } \\
\text { reflux followed by the increase } \\
\text { in temperature up to } 80^{\circ} \mathrm{C} \text { and } \\
\text { remained same for } \\
4 \mathrm{~h} \text {. The gel was formed by } \\
\text { heating up to } 100{ }^{\circ} \mathrm{C} \text { and finally } \\
\text { dried. }\end{array}$ & $\begin{array}{c}\text { Amorphous } \\
\mathrm{SiO}_{2} \text { loaded } \\
\text { with } 10 \mathrm{wt} . \% \text { of } \\
\text { sulfonic groups }\end{array}$ & Soybean oil & $\begin{array}{l}\text { Substrate/tert- } \\
\text { butyl-methyl } \\
\text { ether:1/10, } \\
\text { catalyst: } 1 \text { wt. } \% \text {, } \\
\text { time: -, } \\
\text { temperature: -, } \\
\text { mic. power: } \\
20 \mathrm{~W}\end{array}$ & - & [56] \\
\hline $\begin{array}{l}\text { Heterogeneous } \\
\text { base catalyst- } \\
\text { calcium } \\
\text { diglyceroxide }\end{array}$ & $\begin{array}{l}\text { A specific quantity of calcined } \\
\mathrm{CaO} \text { was put into the glass } \\
\text { reactor carrying methanol and } \\
\text { glycerol. The resulting product } \\
\text { was stirred at } 60^{\circ} \mathrm{C} \text { for } 3 \mathrm{~h} \text { that } \\
\text { lead to the formation of } \mathrm{CaDG} \text {. }\end{array}$ & $\begin{array}{c}\text { CaDG as a } \\
\text { heterogeneous } \\
\text { base catalyst }\end{array}$ & $\begin{array}{l}\text { Waste cooking } \\
\text { oil }\end{array}$ & $\begin{array}{c}\text { M/O: } 7.46, \\
\text { catalyst: } \\
1.03 \text { wt. } \% \text {, time: } \\
15 \text { min, } \\
\text { temperature: } \\
62{ }^{\circ} \mathrm{C} \text {, mic. } \\
\text { assisted }\end{array}$ & $Y=94.86$ & [43] \\
\hline $\begin{array}{c}\text { Pork bone } \\
\text { derived natural } \\
\text { hydroxyapatite }\end{array}$ & Calcination at $900{ }^{\circ} \mathrm{C}$ for $2 \mathrm{~h}$ & $\beta-\mathrm{Ca}_{3}\left(\mathrm{PO}_{4}\right)_{2}$ & $\begin{array}{c}\text { Jatropha Curcas } \\
\text { oil }\end{array}$ & $\begin{array}{l}\text { M/O: } 18, \\
\text { catalyst: } 4 \text { wt. } \% \text {, } \\
\text { time: } 5 \mathrm{~min}, \\
\text { temperature: -, } \\
\text { mic. power: } \\
800 \mathrm{~W}\end{array}$ & $C=94$ & [40] \\
\hline $\begin{array}{l}\text { KF-Modified } \\
\text { Natural } \\
\text { Halloysite }\end{array}$ & $\begin{array}{l}\text { The solid reaction method was } \\
\text { applied for the catalyst } \\
\text { synthesis. The mixture of } \\
\text { potassium fluoride and } \\
\text { halloysite was milled and } \\
\text { heated at a specified } \\
\text { temperature for } 2 \mathrm{~h} . \mathrm{KF} \\
\text { content was differed from } \\
5-30 \text { wt. } \% \text { in range. }\end{array}$ & $\begin{array}{c}\text { KF-Modified } \\
\text { Natural } \\
\text { Halloysite }\end{array}$ & Jatropha oil & $\begin{array}{l}\text { M/O: } 8 \text {, } \\
\text { catalyst/oil: } \\
\text { 1/30, time: } \\
30 \text { min, } \\
\text { temperature: -, } \\
\text { mic. assisted }\end{array}$ & $Y=83.77$ & [57] \\
\hline
\end{tabular}


Table 2. Cont.

\begin{tabular}{|c|c|c|c|c|c|c|}
\hline Materials & Catalyst Preparation & Catalyst & Oil Used & $\begin{array}{l}\text { Reaction } \\
\text { Parameters }\end{array}$ & Yield & Ref. \\
\hline Waste eggshells & $\begin{array}{l}\text { The crushed dried eggshells } \\
\text { were heat-treated in a furnace } \\
\text { at a temperature of } \\
900{ }^{\circ} \mathrm{C}-950^{\circ} \mathrm{C} \text { for } 4 \mathrm{~h} \text {. }\end{array}$ & $\mathrm{CaO}$ & $\begin{array}{l}\text { Lagenaria } \\
\text { vulgaris seed } \\
\text { oil }\end{array}$ & $\begin{array}{l}\text { M/O: } 40, \\
\text { catalyst: } 5 \text { wt. } \% \text {, } \\
\text { time: } 40 \text { min, } \\
\text { temperature: } \\
60^{\circ} \mathrm{C} \text {, mic. } \\
\text { assisted }\end{array}$ & $\mathrm{Y}=95.07$ & [58] \\
\hline $\begin{array}{l}\text { Calcium oxide } \\
\text { catalyst }\end{array}$ & $\begin{array}{l}\mathrm{CaCO}_{3} \text { powder was calcined } \\
\text { for } 5 \mathrm{~h} \text { at } 900^{\circ} \mathrm{C} .\end{array}$ & $\mathrm{CaO}$ & $\begin{array}{l}\text { Waste } \\
\text { cotton-seed } \\
\text { cooking oil }\end{array}$ & $\begin{array}{c}\text { M/O: } 9.6, \\
\text { catalyst: } \\
1.33 \text { wt. } \% \text {, time: } \\
9.7 \text { min, } \\
\text { temperature: -, } \\
\text { mic. assisted }\end{array}$ & $Y=89.94$ & [59] \\
\hline $\begin{array}{c}\text { Hydrated } \\
\text { calcined } \\
\text { Cyrtopleura } \\
\text { costata } \\
\text { seashells }\end{array}$ & $\begin{array}{l}\text { Seashells were initially } \\
\text { washed and dried } \\
\text { accompanied by grinding and } \\
\text { then calcined at } 900{ }^{\circ} \mathrm{C} \text { for } 2 \mathrm{~h} \text {, } \\
\text { Hydration was attained by } \\
\text { placing the heat-treated } \\
\text { product into atmosphere for a } \\
\text { week in a humidity-controlled } \\
\text { room. }\end{array}$ & $\mathrm{Ca}(\mathrm{OH})_{2}$ & Palm oil & $\begin{array}{l}\text { M/O: } 9 \text {, } \\
\text { catalyst: } 0.5 \\
\text { wt.\%, time: } \\
10 \text { min, } \\
\text { temperature: -, } \\
\text { mic. assisted }\end{array}$ & $Y=96.7$ & [44] \\
\hline $\begin{array}{l}\text { Nanopowder } \\
\text { calcium oxide }\end{array}$ & $\begin{array}{c}\text { Nano } \mathrm{CaO} \text { (purity: 98\%) were } \\
\text { purchased and applied. }\end{array}$ & Nano-CaO & Soybean oil & $\begin{array}{l}\text { M/O: } 7, \\
\text { catalyst: } 3 \mathrm{wt} . \% \text {, } \\
\text { time: } 60 \mathrm{~min}, \\
\text { temperature: } \\
60^{\circ} \mathrm{C} \text {, mic. } \\
\text { assisted }\end{array}$ & $Y=96.6$ & [41] \\
\hline $\begin{array}{l}\text { Diphenyla- } \\
\text { mmonium salt } \\
\text { catalysts }\end{array}$ & & $\begin{array}{l}\text { Dipheny- } \\
\text { lammonium } \\
\text { salt }\end{array}$ & Corn oil & $\begin{array}{l}\text { M/O: } 5 / 2 \\
\text { (g/g), catalyst: } \\
20 \text { mol\%, time: } \\
20 \text { min, } \\
\text { temperature: } \\
150^{\circ} \mathrm{C} \text {, mic. } \\
\text { assisted }\end{array}$ & $Y=100$ & [60] \\
\hline $\begin{array}{l}\text { Microwave } \\
\text { Absorption } \\
\text { Catalysts }\end{array}$ & $\begin{array}{c}\mathrm{H}_{2} \mathrm{SO}_{4} / \mathrm{C} \text { catalyst was } \\
\text { synthesised via impregnation } \\
\text { method }\end{array}$ & $\begin{array}{c}\mathrm{H}_{2} \mathrm{SO}_{4} / \mathrm{C} \\
\text { catalyst }\end{array}$ & Castor oil & $\begin{array}{c}\mathrm{M} / \mathrm{O}: 12, \\
\text { catalyst: } 5 \mathrm{wt} \%, \\
55 \mathrm{wt} \% \mathrm{H}_{2} \mathrm{SO}_{4} \\
\text { time: } 60 \mathrm{~min}, \\
\text { temperature: } \\
65^{\circ} \mathrm{C} \text {, mic. } \\
\text { assisted } \\
\end{array}$ & $Y=94$ & [61] \\
\hline $\begin{array}{l}\text { Acidic silica gel } \\
\text { as catalyst }\end{array}$ & $\begin{array}{l}\text { A specified quantity of silica } \\
\text { and sulphuric acid were mixed } \\
\text { and agitated at ambient } \\
\text { temperature for } 30 \mathrm{~min} \text {. The } \\
\text { resulting solid was strained } \\
\text { and finally dried and stored in } \\
\text { a desiccator }\end{array}$ & $\begin{array}{c}\mathrm{H}_{2} \mathrm{SO}_{4} \\
\text { immobilised in } \\
\mathrm{SiO}_{2}\end{array}$ & Castor oil & $\begin{array}{l}\text { M/O: } 6 \text {, } \\
\text { catalyst: } \\
10 \text { wt. } \% \text {, time: } \\
30 \text { min, } \\
\text { temperature: } \\
60^{\circ} \mathrm{C} \text {, mic. } \\
\text { assisted }\end{array}$ & $C=95 \%$ & [62] \\
\hline $\begin{array}{l}\text { Waste Cement } \\
\text { Clinker } \\
\text { Catalyst }\end{array}$ & $\begin{array}{c}\text { Calcinated clinker is utilised to } \\
\text { generate a limestone-based } \\
\text { catalyst }\end{array}$ & $\begin{array}{l}\mathrm{CaO}, \text { Silica, } \\
\text { Alumina, Iron } \\
\text { oxide }\end{array}$ & Rubber seed oil & $\begin{array}{l}\text { M/O: } 5 \text {, } \\
\text { catalyst: } 6 \mathrm{wt} . \% \text {, } \\
\text { time: } 60 \mathrm{~min}, \\
\text { temperature: } \\
60^{\circ} \mathrm{C} \text {, mic. } \\
\text { assisted }\end{array}$ & $C=96.8$ & [63] \\
\hline
\end{tabular}


Table 2. Cont.

\begin{tabular}{|c|c|c|c|c|c|c|}
\hline Materials & Catalyst Preparation & Catalyst & Oil Used & $\begin{array}{c}\text { Reaction } \\
\text { Parameters }\end{array}$ & Yield & Ref. \\
\hline $\begin{array}{l}\text { KSF montmoril- } \\
\text { lonite }\end{array}$ & $\begin{array}{l}\text { Catalysts have been purchased } \\
\text { from Aldrich }\end{array}$ & & Rape oil & $\begin{array}{c}\text { M/O: } 9, \\
\text { catalyst: } \\
10 \text { wt.\%, time: } \\
60 \text { min, } \\
\text { temperature: } \\
170^{\circ} \mathrm{C} \text {, mic. } \\
\text { assisted }\end{array}$ & $\mathrm{Y}=51$ & [64] \\
\hline $\begin{array}{l}\text { Aminophosphonic } \\
\text { acid resin D418 }\end{array}$ & $\begin{array}{l}\text { Aminophosphonic acid resin } \\
\text { D418 was purchased and used. }\end{array}$ & $\begin{array}{l}\text { Macroporous } \\
\text { styrene chelate } \\
\text { resin, with } \\
-\mathrm{NHCH}_{2} \mathrm{PO}_{3} \mathrm{H}_{2} \\
\text { functional } \\
\text { groups }\end{array}$ & $\begin{array}{l}\text { Free fatty acid } \\
\text { stearic acid }\end{array}$ & $\begin{array}{l}\text { M/O: } 11, \\
\text { catalyst: } 9 \text { wt. } \% \text {, } \\
\text { time: } 7 \mathrm{~h}, \\
\text { temperature: } \\
80^{\circ} \mathrm{C} \text {, mic. } \\
\text { assisted }\end{array}$ & $Y>90$ & [65] \\
\hline $\begin{array}{l}\text { Calcium oxide } \\
(\mathrm{CaO}) \text { loaded } \\
\text { on zeolite }\end{array}$ & $\begin{array}{l}\text { The manufacturing of an } \\
\text { active solid catalyst via CaO } \\
\text { loaded on high silica zeolite } \\
\text { employing impregnation } \\
\text { method }\end{array}$ & $\begin{array}{c}35 \% \\
\mathrm{CaO} / \text { zeolite }\end{array}$ & Waste lard fat & $\begin{array}{l}\text { M/O: } 30, \\
\text { catalyst: } 8 \mathrm{w} / v, \\
\text { time: } 1.25 \mathrm{~min}, \\
\text { temperature: }- \text {, } \\
\text { mic. power: } \\
595 \mathrm{~W}\end{array}$ & $\mathrm{Y}=90.89$ & [66] \\
\hline $\begin{array}{l}\mathrm{K}_{2} \mathrm{CO}_{3} / \mathrm{Al}_{2} \mathrm{O} \\
\text { catalyst }\end{array}$ & $\begin{array}{c}\text { The supported catalysts } \\
\mathrm{K}_{2} \mathrm{CO}_{3} / \mathrm{Al}_{2} \mathrm{O}_{3} \text {, was attained } \\
\text { via sedimentation } \\
\text { subsequently, the calcination } \\
\text { of the catalyst active mass. }\end{array}$ & $\begin{array}{c}5 \% \\
\mathrm{~K}_{2} \mathrm{CO}_{3} / \mathrm{Al}_{2} \mathrm{O}\end{array}$ & Sunflower oil & $\begin{array}{c}\text { M/O: } 16, \\
\text { catalyst: } \\
2.5 \text { wt. } \% \text {, time: } \\
30 \text { min, } \\
\text { temperature: } \\
70{ }^{\circ} \mathrm{C} \text {, mic. } \\
\text { assisted }\end{array}$ & $C>60$ & [67] \\
\hline $\begin{array}{l}\text { Graphene } \\
\text { oxide-based } \\
\text { catalyst }\end{array}$ & $\begin{array}{l}\text { Graphene oxide was } \\
\text { synthesised from graphite } \\
\text { powders through modified } \\
\text { Hummer's method. The } \\
\text { SiC-NaOH/GO was } \\
\text { synthesised by in-situ } \\
\text { impregnation method. }\end{array}$ & $\begin{array}{l}\mathrm{SiC}-\mathrm{NaOH} / \mathrm{GO} \\
\text { catalyst }\end{array}$ & $\begin{array}{c}\text { Chlorella } \\
\text { vulgaris lipid }\end{array}$ & $\begin{array}{l}\text { M/O: } 48, \\
\text { catalyst: } 4 \text { wt. } \% \text {, } \\
\text { time: } 5 \text { min, } \\
\text { temperature: } \\
85^{\circ} \mathrm{C} \text {, mic. } \\
\text { assisted }\end{array}$ & $Y=81$ & [68] \\
\hline $\begin{array}{l}\text { Elephant-ear } \\
\text { tree pod husk }\end{array}$ & $\begin{array}{l}\text { The dried pods were } \\
\text { handpicked, and seeds were } \\
\text { separated manually parting } \\
\text { the sticky husk behind. One } \\
\text { part of the husks was } \\
\text { powdered, as the other portion } \\
\text { was charred to ash in air. After } \\
\text { that, part of the ash was } \\
\text { exposed to calcination } \\
\text { between } 300 \text { and } 1100{ }^{\circ} \mathrm{C} \text { for } \\
4 \mathrm{~h} \text { using a muffle furnace. }\end{array}$ & $\begin{array}{c}\mathrm{K}, \mathrm{Mg}, \mathrm{Ca} \text { and } \\
\mathrm{Fe}\end{array}$ & $\begin{array}{c}\text { Esterified oil } \\
\text { mix of neem } \\
\text { and rubber seed } \\
\text { oil }\end{array}$ & $\begin{array}{l}\text { M/O: } 11.44, \\
\text { catalyst: } \\
2.96 \text { wt.\%, time: } \\
5.88 \text { min, } \\
\text { temperature: -, } \\
\text { mic. power: } \\
150 \mathrm{~W}\end{array}$ & $Y=98.77$ & [69] \\
\hline $\begin{array}{c}\mathrm{ZnO} / \mathrm{La}_{2} \mathrm{O}_{2} \mathrm{CO}_{3} \\
\text { layered } \\
\text { composite }\end{array}$ & $\begin{array}{l}\text { The zinc-lanthanum-mixed } \\
\text { oxide catalyst was synthesised } \\
\text { through an in-situ } \\
\text { precipitation technique and } \\
\text { later calcined at } 550^{\circ} \mathrm{C} \text { for } 6 \mathrm{~h}\end{array}$ & $\mathrm{ZnO} / \mathrm{La}_{2} \mathrm{O}_{2} \mathrm{CO}_{3}$ & Canola oil & $\begin{array}{l}\text { M/O: } 12, \\
\text { catalyst: } \\
<1 \text { wt. } \% \text {, time: } \\
5 \text { min, } \\
\text { temperature: } \\
85^{\circ} \mathrm{C} \text {, mic. } \\
\text { assisted }\end{array}$ & $Y>95$ & [70] \\
\hline
\end{tabular}




\section{Special Thermal Effects of Microwaves on Solid Catalysts}

The dielectric materials captivate microwave energy and convert it into heat energy which is appeared as the total loss of microwave energy in materials. This phenomenon is called the thermal effect of the microwave. Figure 2 shows the special thermal effect, also called hot spot, uncovered by various investigators [71-73].

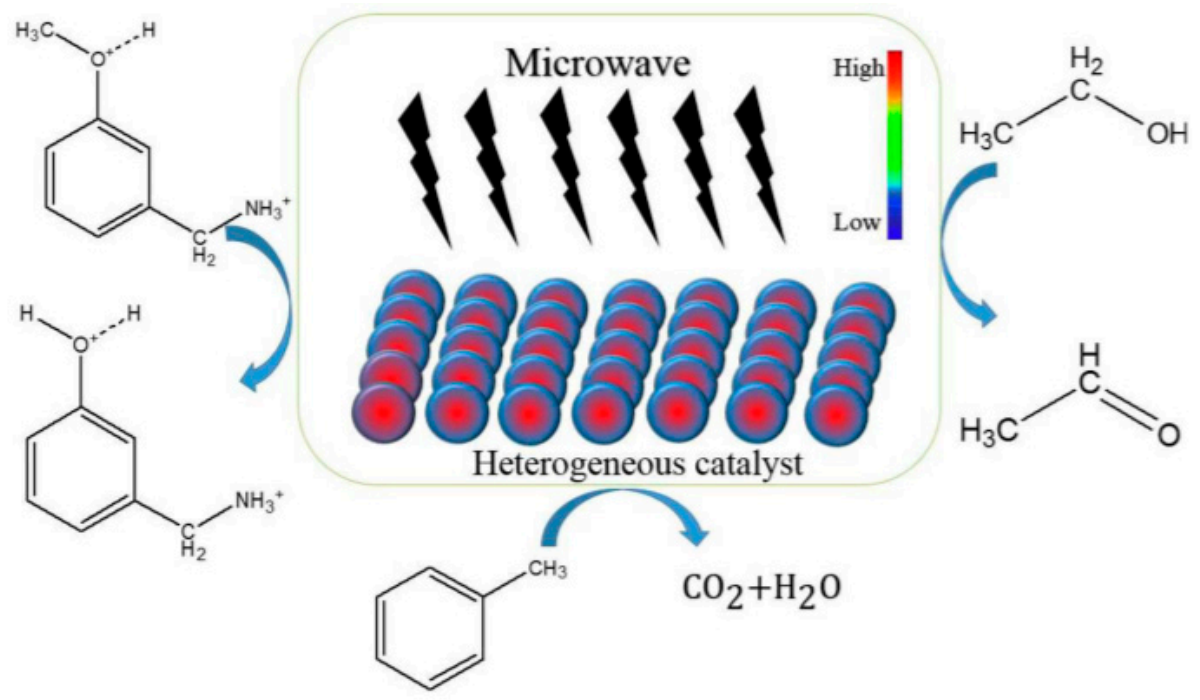

Figure 2. Thermal effect mechanism of microwave on heterogeneous catalysts.

Moreover, two mechanisms such as conduction in material and polarisation, are involved in dielectric heating. In conduction, variation in the electric field would oscillate the mobile charged particles or ions, which results in the electric current generation. Heat is generated owing to the internal resistance to this current provoked by the collision of molecules and charged particles. The conduction of charged ions moving randomly in the mixture may also lead to generating heat. If these ions are in a space circumscribed by other molecules and cannot freely couple with the varying electric field, the charge begins to accumulate, and the energy is dissipated as heat [11].

Whereas, in the case of polarisation, the displacement of charged particles from their equilibrium positions occurs within the dielectric material owing to the electric field, inducing dipoles. These dipoles begin to rotate with high frequency upon responding to the applied electric field may generate heat and friction. Polar materials have permanent dipoles that also result in polarisation [11].

Microwave-assisted biodiesel synthesis has been studied with various homogeneous and heterogeneous catalysts by Nayak and his coworkers [74]. The major benefit coupled with the usage of heterogeneous catalysts is their simple separation from the product and reclaim in comparison with homogeneous catalysts. There exists a number of acid or base heterogeneous catalysts synthesised for biodiesel generation. The multiple selectivities of solid catalysts permit the choice of the appropriate catalyst for the reaction [72]. For example, Yuan et al. [61] explored the use of $\mathrm{H}_{2} \mathrm{SO}_{4} / \mathrm{C}$ solid acid catalyst for the transesterification reaction in microwave irradiation. Both sulfuric acid and activated carbon possess high dielectric properties and, therefore, absorb microwaves. Owing to this, microwave hotspots are generated on $\mathrm{H}_{2} \mathrm{SO}_{4} / \mathrm{C}$ solid acid catalyst in the presence of microwaves which indicates that the temperature of the catalyst would be greater than that of liquid. The elevated temperature from microwave hotspots would offset the activity reduction owing to mass transfer resistance resulted in better catalytic activity. Moreover, in the case of solid catalysts, both internal and external surfaces are simultaneously available for reaction. The endothermal reaction favors high temperature as in the case of transesterification using $\mathrm{H}_{2} \mathrm{SO}_{4} / \mathrm{C}$ solid acid catalyst for biodiesel generation, therefore, yield is improved under 
microwave heating. In this case, a maximum yield of $94 \%$ was achieved under microwave heating compared with conventional heating.

Dipolar polarisation, interfacial polarisation and ionic conduction mechanisms are responsible for microwave energy transfer into microwave absorbing materials [75]. Another explanation stated by the researchers that methanol utilised in transesterification reaction holds a robust microwave absorption property, and hydroxyl groups connected with long-chain molecules act as if fastened to a stagnant raft, with the more localised rotations governing the microwave spectrum and ensuing a molecular level localised superheating, subsequently a substantial drop in activation energy that supports in accelerating the reaction [76,77]. Marwan et al. [44] described that the temperature attained at the catalyst surface could be much greater than the bulk temperature, which increased the biodiesel yield due to the localised superheating.

\section{Optimisation of Reaction Parameters for Microwave Mediated Heterogeneously Catalysed Transesterification}

The optimisation of reaction variables such as alcohol/oil ratio, catalyst weight percent, reaction time, temperature and microwave power etc., are still required for maximising the biodiesel yield under microwave irradiation, similar to the conventional heating scenario. It has been reported that for conventional heating, the optimised reaction parameters for four heterogeneous catalysts including $\mathrm{BaO}, \mathrm{SrO}, \mathrm{CaO}$ and $\mathrm{MgO}$ were found to be methanol/oil: 9:1, 12:1, 15:1 and 15:1 respectively, the reaction temperatures of 100, 60, 100, $80{ }^{\circ} \mathrm{C}$, respectively and catalyst loading of $1 \%, 0.5 \%, 0.5 \%$ and $1 \%(\mathrm{wt})$, respectively [78]. On another side, the most favourable reaction conditions for microwave mediated reaction of $\mathrm{C}$. sativa oil in the presence of $\mathrm{BaO}$ and $\mathrm{SrO}$ catalysts were observed to be methanol/oil: 9:1 and catalyst loading of $1.5 \%$ and $2 \%$, respectively. While the reaction times have been significantly dropped for the case of microwave heating using the same catalysts as compared to the standard heating (Figure 3) [42]. However, it seems noteworthy that a higher catalyst amount is required for getting higher biodiesel yield in such a short reaction time. Nowadays, different optimisation techniques such as RSM, ANN, ANFIS, DoE, Taguchi method and some hybrid approaches such as SVM-RSM and ELM-RSM have also been employed for the optimisation of reaction parameters [22,23,79,80].
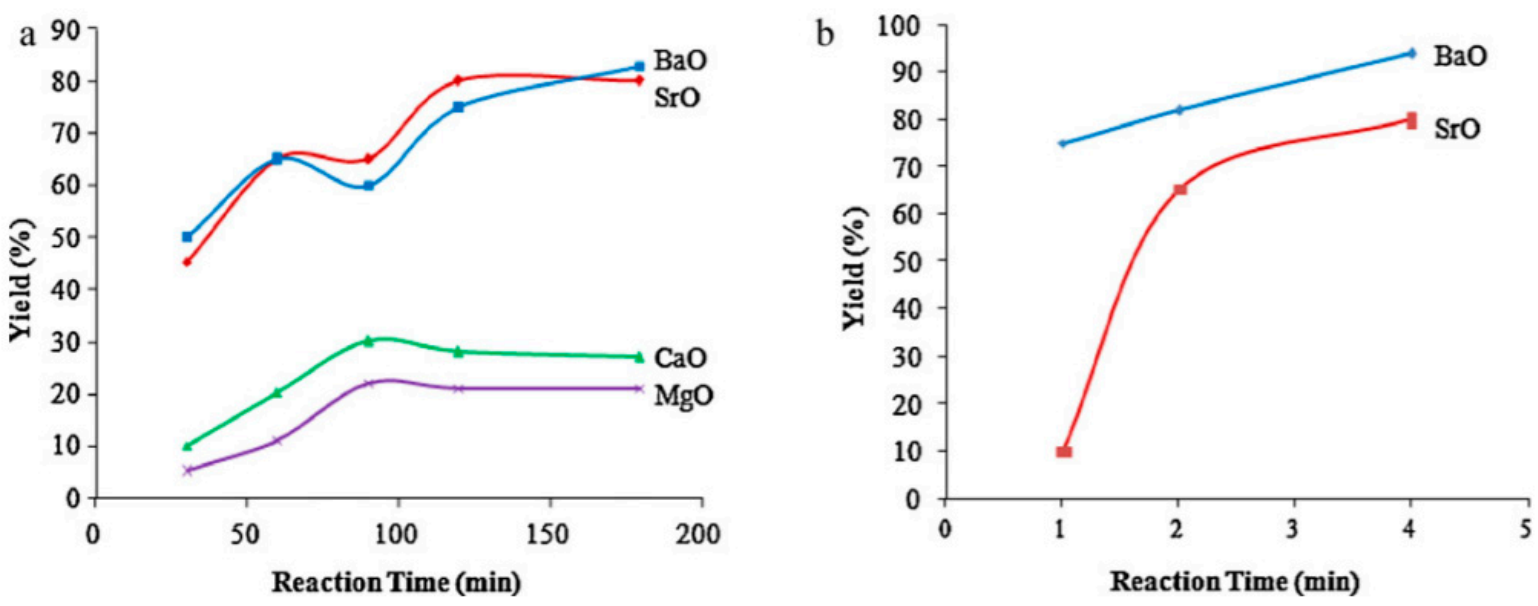

Figure 3. FAME yield vs. reaction time for same catalysts under (a) Normal heating, (b) microwave heating [42] (Licence number: 5196451338660).

\section{Conclusions}

With technological advancement, it has become necessary to adopt cutting-edge technologies to make the overall process more sustainable and time-efficient, particularly in renewable fuels production, such as biodiesel, which is required to avoid the threats of oil reserve depletion and resulting environmental damage. Many studies have been conducted 
in recent years on biodiesel production from diverse feedstocks utilising heterogeneous catalysts and traditional heating techniques. Currently, research is being devoted to implementing microwave heating technology for biodiesel synthesis employing heterogeneous catalysts to improve the process's efficiency. This study focuses on the research done to date in microwave aided heterogeneously catalysed biodiesel generation. It examines the phenomena of microwave heating, synthesis strategies for heterogeneous catalysts, microwave mediated transesterification process employing solid catalysts, specific thermal effects of microwaves, and parametric optimisation under microwave heating. The application of microwave technology for biodiesel generation using heterogeneous catalysts has appeared to be quite efficient with respect to biodiesel yield, catalytic activity, reaction time and energy efficiency. It strongly suggests that the application of microwave technology on the heterogeneously catalysed transesterification process significantly reduces the reaction times with better catalytic activity compared to conventional heating. Although microwave mediated biodiesel production is considered fast and energy-efficient, but the major downside associated with it is its lower penetration depth that is a few centimetres. It displays the instant loss in microwave intensity, and this problem may amplify while scaling up the batch process. This issue is required to be rectified, or continuous mode can be adopted using film type reactor to overcome this problem. Moreover, there are other risks such as spark ignition, high-temperature zones at edges, microwave leakages etc., which must be tackled for large scale adoption of this technology. If the mentioned issues are properly managed, commercial adoption of microwave technology would be quite helpful in satisfying the rising demands of biofuels.

Author Contributions: Conceptualisation, H.M.K. and T.I.; methodology, H.M.K. and M.A.M.; validation, M.A.M., I.V. and M.E.M.S.; writing—original draft preparation, H.M.K. and I.M.R.F.; writing-review and editing, I.M.R.F.; supervision, M.A.M. and I.M.R.F. All authors have read and agreed to the published version of the manuscript.

Funding: This research received no external funding.

Institutional Review Board Statement: Not applicable.

Informed Consent Statement: Not applicable.

Data Availability Statement: No new data were created or analyzed in this study. Data sharing is not applicable to this article.

Conflicts of Interest: The authors declare no conflict of interest.

\section{References}

1. Khan, H.M.; Iqbal, T.; Yasin, S.; Ali, C.H.; Abbas, M.M.; Jamil, M.A.; Hussain, A.; M Soudagar, M.E.; Rahman, M.M. Application of Agricultural Waste as Heterogeneous Catalysts for Biodiesel Production. Catalysts 2021, 11, 1215. [CrossRef]

2. Soudagar, M.E.M.; Khan, H.M.; Khan, T.; Razzaq, L.; Asif, T.; Mujtaba, M.; Hussain, A.; Farooq, M.; Ahmed, W.; Shahapurkar, K. Experimental analysis of engine performance and exhaust pollutant on a single-cylinder diesel engine operated using moringa oleifera biodiesel. Appl. Sci. 2021, 11, 7071. [CrossRef]

3. Su, G.; Ong, H.C.; Ibrahim, S.; Fattah, I.M.R.; Mofijur, M.; Chong, C.T. Valorisation of medical waste through pyrolysis for a cleaner environment: Progress and challenges. Environ. Pollut. 2021, 279, 116934. [CrossRef]

4. Su, G.; Ong, H.C.; Fattah, I.M.R.; Ok, Y.S.; Jang, J.-H.; Wang, C.-T. State-of-the-art of the pyrolysis and co-pyrolysis of food waste: Progress and challenges. Sci. Total Environ. 2021, 151170. [CrossRef]

5. Salaheldeen, M.; Mariod, A.A.; Aroua, M.K.; Rahman, S.M.A.; Soudagar, M.E.M.; Fattah, I.M.R. Current State and Perspectives on Transesterification of Triglycerides for Biodiesel Production. Catalysts 2021, 11, 1121. [CrossRef]

6. Fattah, I.M.R.; Ong, H.C.; Mahlia, T.M.I.; Mofijur, M.; Silitonga, A.S.; Rahman, S.M.A.; Ahmad, A. State of the Art of Catalysts for Biodiesel Production. Front. Energy Res. 2020, 8, 101. [CrossRef]

7. Lv, L.; Dai, L.; Du, W.; Liu, D. Progress in Enzymatic Biodiesel Production and Commercialization. Processes 2021, 9, 355. [CrossRef]

8. Luque, R.; Lovett, J.C.; Datta, B.; Clancy, J.; Campelo, J.M.; Romero, A.A. Biodiesel as feasible petrol fuel replacement: A multidisciplinary overview. Energy Environ. Sci. 2010, 3, 1706-1721. [CrossRef]

9. Khan, H.M.; Iqbal, T.; Yasin, S.; Irfan, M.; Kazmi, M.; Fayaz, H.; Mujtaba, M.; Ali, C.H.; Kalam, M.; Soudagar, M.E.M. Production and utilization aspects of waste cooking oil based biodiesel in Pakistan. Alex. Eng. J. 2021, 60, 5831-5849. [CrossRef] 
10. Sharma, M.P. Selection of potential oils for biodiesel production. Renew. Sustain. Energy Rev. 2016, 56, 1129-1138.

11. Muley, P.D.; Wang, Y.; Hu, J.; Shekhawat, D. Microwave-Assisted Heterogeneous catalysis. In Catalysis: Volume 33; Spivey, J., Han, Y.-F.S., Dushyant, S., Eds.; Royal Society of Chemistry: Piccadilly, UK, 2021; Volume 33, pp. 1-37.

12. Avhad, M.; Marchetti, J. Innovation in solid heterogeneous catalysis for the generation of economically viable and ecofriendly biodiesel: A review. Catal. Rev. 2016, 58, 157-208. [CrossRef]

13. Coman, S.M.; Parvulescu, V.I. Heterogeneous Catalysis for Biodiesel Production. In The Role of Catalysis for the Sustainable Production of Bio-Fuels and Bio-Chemicals; Elsevier: Amsterdam, The Netherlands, 2013; pp. 93-136.

14. Sahu, G.; Gupta, N.K.; Kotha, A.; Saha, S.; Datta, S.; Chavan, P.; Kumari, N.; Dutta, P. A review on biodiesel production through heterogeneous catalysis route. ChemBioEng Rev. 2018, 5, 231-252. [CrossRef]

15. Ruhul, A.M.; Kalam, M.A.; Masjuki, H.H.; Fattah, I.M.R.; Reham, S.S.; Rashed, M.M. State of the art of biodiesel production processes: A review of the heterogeneous catalyst. RSC Adv. 2015, 5, 101023-101044. [CrossRef]

16. Ong, H.C.; Tiong, Y.W.; Goh, B.H.H.; Gan, Y.Y.; Mofijur, M.; Fattah, I.M.R.; Chong, C.T.; Alam, M.A.; Lee, H.V.; Silitonga, A.S.; et al. Recent advances in biodiesel production from agricultural products and microalgae using ionic liquids: Opportunities and challenges. Energy Convers. Manag. 2021, 228, 113647. [CrossRef]

17. Kremsner, J.M.; Kappe, C.O. Microwave-Assisted Organic Synthesis in Near-Critical Water at $300{ }^{\circ} \mathrm{C}-$ A Proof-of-Concept Study; Wiley Online Library: Hoboken, NJ, USA, 2005.

18. Lidström, P.; Tierney, J.; Watheyb, B.; Westmana, J. Microwave assisted organic synthesisĐa review. Tetrahedron 2001, 57, 9225-9283. [CrossRef]

19. Chen, C.-C.; Reddy, P.M.; Devi, C.S.; Chang, P.-C.; Ho, Y.-P. Study of microwave effects on the lipase-catalyzed hydrolysis. Enzym. Microb. Technol. 2016, 82, 164-172. [CrossRef]

20. Nomanbhay, S.; Ong, M.Y. A review of microwave-assisted reactions for biodiesel production. Bioengineering 2017, 4, 57. [CrossRef]

21. Refaat, A. Different techniques for the production of biodiesel from waste vegetable oil. Int. J. Environ. Sci. Technol. 2010, 7, 183-213. [CrossRef]

22. Ardabili, S.F.; Najafi, B.; Alizamir, M.; Mosavi, A.; Shamshirband, S.; Rabczuk, T. Using SVM-RSM and ELM-RSM approaches for optimizing the production process of methyl and ethyl esters. Energies 2018, 11, 2889. [CrossRef]

23. Najafi, B.; Ardabili, S.F.; Shamshirband, S.; Chau, K.-W.; Rabczuk, T. Application of ANNs, ANFIS and RSM to estimating and optimizing the parameters that affect the yield and cost of biodiesel production. Eng. Appl. Comput. Fluid Mech. 2018, 12, 611-624. [CrossRef]

24. Zhang, Y. Preparation of heterogeneous catalysts based on CWAO technology. J. Phys. Conf. Ser. 2020, 1549, 032052. [CrossRef]

25. Hussain, F.; Alshahrani, S.; Abbas, M.M.; Khan, H.M.; Jamil, A.; Yaqoob, H.; Soudagar, M.E.M.; Imran, M.; Ahmad, M.; Munir, M. Waste Animal Bones as Catalysts for Biodiesel Production; A Mini Review. Catalysts 2021, 11, 630. [CrossRef]

26. Lee, A.F.; Bennett, J.A.; Manayil, J.C.; Wilson, K. Heterogeneous catalysis for sustainable biodiesel production via esterification and transesterification. Chem. Soc. Rev. 2014, 43, 7887-7916. [CrossRef] [PubMed]

27. Dai, Y.-M.; Chen, K.-T.; Chen, C.-C. Study of the microwave lipid extraction from microalgae for biodiesel production. Chem. Eng. J. 2014, 250, 267-273. [CrossRef]

28. Dai, Y.-M.; Wu, J.-S.; Chen, C.-C.; Chen, K.-T. Evaluating the optimum operating parameters on transesterification reaction for biodiesel production over a $\mathrm{LiAlO}_{2}$ catalyst. Chem. Eng. J. 2015, 280, 370-376. [CrossRef]

29. Wang, J.-X.; Chen, K.-T.; Wu, J.-S.; Wang, P.-H.; Huang, S.-T.; Chen, C.-C. Production of biodiesel through transesterification of soybean oil using lithium orthosilicate solid catalyst. Fuel Process. Technol. 2012, 104, 167-173. [CrossRef]

30. Kim, M.Y.; Lee, K.; Choi, M. Cooperative effects of secondary mesoporosity and acid site location in Pt/SAPO-11 on n-dodecane hydroisomerization selectivity. J. Catal. 2014, 319, 232-238. [CrossRef]

31. Wang, Y.; Chen, B.-b.; Crocker, M.; Zhang, Y.-j.; Zhu, X.-b.; Shi, C. Understanding on the origins of hydroxyapatite stabilized gold nanoparticles as high-efficiency catalysts for formaldehyde and benzene oxidation. Catal. Commun. 2015, 59, 195-200. [CrossRef]

32. Yang, Z.; Zhang, Y.; Ding, W.; Zhang, Y.; Shen, P.; Zhou, Y.; Liu, Y.; Huang, S.; Lu, X. Hydrogen production from coke oven gas over $\mathrm{LiNi} / \gamma-\mathrm{Al}_{2} \mathrm{O}_{3}$ catalyst modified by rare earth metal oxide in a membrane reactor. J. Nat. Gas Chem. 2009, 18, 407-414. [CrossRef]

33. Kuo, C.-W.; Chen, B.-K.; Tseng, Y.-H.; Hsieh, T.-H.; Ho, K.-S.; Wu, T.-Y.; Chen, H.-R. A comparative study of poly (acrylic acid) and poly (styrenesulfonic acid) doped into polyaniline as platinum catalyst support for methanol electro-oxidation. J. Taiwan Inst. Chem. Eng. 2012, 43, 798-805. [CrossRef]

34. Awogbemi, O.; Von Kallon, D.; Aigbodion, V.S. Trends in the development and utilization of agricultural wastes as heterogeneous catalyst for biodiesel production. J. Energy Inst. 2021, 98, 244-258. [CrossRef]

35. Khedri, B.; Mostafaei, M.; Ardebili, S.M.S. A review on microwave-assisted biodiesel production. Energy Sources Part A Recovery Util. Environ. Eff. 2019, 41, 2377-2395. [CrossRef]

36. Khemthong, P.; Luadthong, C.; Nualpaeng, W.; Changsuwan, P.; Tongprem, P.; Viriya-Empikul, N.; Faungnawakij, K. Industrial eggshell wastes as the heterogeneous catalysts for microwave-assisted biodiesel production. Catal. Today 2012, 190, 112-116. [CrossRef]

37. Rocha, P.D.; Oliveira, L.S.; Franca, A.S. Sulfonated activated carbon from corn cobs as heterogeneous catalysts for biodiesel production using microwave-assisted transesterification. Renew. Energy 2019, 143, 1710-1716. [CrossRef] 
38. Zhang, S.; Zu, Y.-G.; Fu, Y.-J.; Luo, M.; Zhang, D.-Y.; Efferth, T. Rapid microwave-assisted transesterification of yellow horn oil to biodiesel using a heteropolyacid solid catalyst. Bioresour. Technol. 2010, 101, 931-936. [CrossRef]

39. Singh, V.; Sharma, Y.C. Low cost guinea fowl bone derived recyclable heterogeneous catalyst for microwave assisted transesterification of Annona squamosa L. seed oil. Energy Convers. Manag. 2017, 138, 627-637. [CrossRef]

40. Buasri, A.; Inkaew, T.; Kodephun, L.; Yenying, W.; Loryuenyong, V. Natural hydroxyapatite (NHAp) derived from pork bone as a renewable catalyst for biodiesel production via microwave irradiation. Key Eng. Mater. 2015, 569, 216-220. [CrossRef]

41. Hsiao, M.-C.; Lin, C.-C.; Chang, Y.-H. Microwave irradiation-assisted transesterification of soybean oil to biodiesel catalyzed by nanopowder calcium oxide. Fuel 2011, 90, 1963-1967. [CrossRef]

42. Patil, P.; Gude, V.G.; Pinappu, S.; Deng, S. Transesterification kinetics of Camelina sativa oil on metal oxide catalysts under conventional and microwave heating conditions. Chem. Eng. J. 2011, 168, 1296-1300. [CrossRef]

43. Gupta, A.R.; Rathod, V.K. Calcium diglyceroxide catalyzed biodiesel production from waste cooking oil in the presence of microwave: Optimization and kinetic studies. Renew. Energy 2018, 121, 757-767. [CrossRef]

44. Indarti, E. Hydrated calcined Cyrtopleura costata seashells as an effective solid catalyst for microwave-assisted preparation of palm oil biodiesel. Energy Convers. Manag. 2016, 117, 319-325.

45. Buasri, A.; Rattanapan, T.; Boonrin, C.; Wechayan, C.; Loryuenyong, V. Oyster and Pyramidella shells as heterogeneous catalysts for the microwave-assisted biodiesel production from Jatropha curcas oil. J. Chem. 2015, 2015, 578625. [CrossRef]

46. Fatimah, I.; Rubiyanto, D.; Taushiyah, A.; Najah, F.B.; Azmi, U.; Sim, Y.-L. Use of ZrO2 supported on bamboo leaf ash as a heterogeneous catalyst in microwave-assisted biodiesel conversion. Sustain. Chem. Pharm. 2019, 12, 100129. [CrossRef]

47. Chellappan, S.; Aparna, K.; Chingakham, C.; Sajith, V.; Nair, V. Microwave assisted biodiesel production using a novel Brønsted acid catalyst based on nanomagnetic biocomposite. Fuel 2019, 246, 268-276. [CrossRef]

48. Fatimah, I.; Rubiyanto, D.; Nugraha, J. Preparation, characterization, and modelling activity of potassium flouride modified hydrotalcite for microwave assisted biodiesel conversion. Sustain. Chem. Pharm. 2018, 8, 63-70. [CrossRef]

49. Liao, C.-C.; Chung, T.-W. Optimization of process conditions using response surface methodology for the microwave-assisted transesterification of Jatropha oil with KOH impregnated CaO as catalyst. Chem. Eng. Res. Des. 2013, 91, 2457-2464. [CrossRef]

50. Nazir, M.H.; Ayoub, M.; Zahid, I.; Shamsuddin, R.B.; Yusup, S.; Ameen, M.; Qadeer, M.U. Development of lignin based heterogeneous solid acid catalyst derived from sugarcane bagasse for microwave assisted-transesterification of waste cooking oil. Biomass Bioenerg. 2021, 146, 105978. [CrossRef]

51. Ye, B.; Qiu, F.; Sun, C.; Li, Y.; Yang, D. Transesterification of Soybean Oil to Biodiesel in a Microwave-Assisted Heterogeneous Catalytic System. Chem. Eng. Technol. 2014, 37, 283-292. [CrossRef]

52. Lokman, I.M.; Rashid, U.; Taufiq-Yap, Y.H. Microwave-Assisted Methyl Ester Production from Palm Fatty Acid Distillate over a Heterogeneous Carbon-Based Solid Acid Catalyst. Chem. Eng. Technol. 2015, 38, 1837-1844. [CrossRef]

53. Varol, P.M.; Çakan, A.; Kiren, B.; Ayas, N. Microwave-assisted catalytic transesterification of soybean oil using $\mathrm{KOH} / \gamma-\mathrm{Al}_{2} \mathrm{O}_{3}$. Biomass Convers. Biorefinery 2021, 1-13. [CrossRef]

54. Wahidin, S.; Idris, A.; Yusof, N.M.; Kamis, N.H.H.; Shaleh, S.R.M. Optimization of the ionic liquid-microwave assisted one-step biodiesel production process from wet microalgal biomass. Energy Convers. Manag. 2018, 171, 1397-1404. [CrossRef]

55. Lee, H.; Wu, W.-H.; Chen, B.-H.; Liao, J.-D. Heterogeneous Catalysts Using Strontium Oxide Agglomerates Depositing upon Titanium Plate for Enhancing Biodiesel Production. Catalysts 2021, 11, 30. [CrossRef]

56. Drago, C.; Liotta, L.F.; La Parola, V.; Testa, M.L.; Nicolosi, G. One-pot microwave assisted catalytic transformation of vegetable oil into glycerol-free biodiesel. Fuel 2013, 113, 707-711. [CrossRef]

57. Fatimah, I.; Yudha, S.P. KF-modified natural halloysite as green catalyst in microwave assisted biodiesel conversion. Energy Procedia 2017, 105, 1796-1805. [CrossRef]

58. Umar, A.; Uba, A.; Mohammed, M.; Almustapha, M.; Muhammad, C.; Sani, J. Microwave assisted biodiesel production from Lagenaria vulgaris seed oil using amberlyst 15 ion exchange resin and eggshell as catalysts. Niger. J. Basic Appl. Sci. 2018, 26, 88-96. [CrossRef]

59. Sharma, A.; Kodgire, P.; Kachhwaha, S.S. Biodiesel production from waste cotton-seed cooking oil using microwave-assisted transesterification: Optimization and kinetic modeling. Renew. Sustain. Energy Rev. 2019, 116, 109394. [CrossRef]

60. Majewski, M.W.; Pollack, S.A.; Curtis-Palmer, V.A. Diphenylammonium salt catalysts for microwave assisted triglyceride transesterification of corn and soybean oil for biodiesel production. Tetrahedron Lett. 2009, 50, 5175-5177. [CrossRef]

61. Yuan, H.; Yang, B.; Zhu, G. Synthesis of biodiesel using microwave absorption catalysts. Energy Fuels 2009, 23, 548-552. [CrossRef]

62. Perin, G.; Álvaro, G.; Westphal, E.; Viana, L.; Jacob, R.; Lenardão, E.; D'Oca, M. Transesterification of castor oil assisted by microwave irradiation. Fuel 2008, 87, 2838-2841. [CrossRef]

63. Hussain, A.; Ali, S.; Ahmed, I.; Gimbun, J.; Albeirutty, M.H.; Rehan, Z.A. Microwave reinforced transesterification of rubber seed oil using waste cement clinker catalyst. Curr. Nanosci. 2016, 12, 576-585. [CrossRef]

64. Mazzocchia, C.; Modica, G.; Kaddouri, A.; Nannicini, R. Fatty acid methyl esters synthesis from triglycerides over heterogeneous catalysts in the presence of microwaves. C. R. Chim. 2004, 7, 601-605. [CrossRef]

65. Liu, W.; Yin, P.; Liu, X.; Chen, W.; Chen, H.; Liu, C.; Qu, R.; Xu, Q. Microwave assisted esterification of free fatty acid over a heterogeneous catalyst for biodiesel production. Energy Convers. Manag. 2013, 76, 1009-1014. [CrossRef]

66. Lawan, I.; Garba, Z.N.; Zhou, W.; Zhang, M.; Yuan, Z. Synergies between the microwave reactor and CaO/zeolite catalyst in waste lard biodiesel production. Renew. Energy 2020, 145, 2550-2560. [CrossRef] 
67. Dall'Oglio, E.L.; Sousa, P.T.d., Jr.; Oliveira, P.T.d.J.; Vasconcelos, L.G.d.; Parizotto, C.A.; Kuhnen, C.A. Use of heterogeneous catalysts in methylic biodiesel production induced by microwave irradiation. Química Nova 2014, 37, 411-417.

68. Loy, A.C.M.; Quitain, A.T.; Lam, M.K.; Yusup, S.; Sasaki, M.; Kida, T. Development of high microwave-absorptive bifunctional graphene oxide-based catalyst for biodiesel production. Energy Convers. Manag. 2019, 180, 1013-1025. [CrossRef]

69. Falowo, O.A.; Oloko-Oba, M.I.; Betiku, E. Biodiesel production intensification via microwave irradiation-assisted transesterification of oil blend using nanoparticles from elephant-ear tree pod husk as a base heterogeneous catalyst. Chem. Eng. Process. Process Intensif. 2019, 140, 157-170. [CrossRef]

70. Jin, L.; Zhang, Y.; Dombrowski, J.P.; Chen, C.-H.; Pravatas, A.; Xu, L.; Perkins, C.; Suib, S.L. ZnO/ $\mathrm{La}_{2} \mathrm{O}_{2} \mathrm{CO}_{3}$ layered composite: A new heterogeneous catalyst for the efficient ultra-fast microwave biofuel production. Appl. Catal. B Environ. 2011, 103, 200-205. [CrossRef]

71. Jiang, S.; Daly, H.; Xiang, H.; Yan, Y.; Zhang, H.; Hardacre, C.; Fan, X. Microwave-assisted catalyst-free hydrolysis of fibrous cellulose for deriving sugars and biochemicals. Front. Chem. Sci. Eng. 2019, 13, 718-726. [CrossRef]

72. Li, H.; Zhang, C.; Pang, C.; Li, X.; Gao, X. The advances in the special microwave effects of the heterogeneous catalytic reactions. Front. Chem. 2020, 8, 355. [CrossRef] [PubMed]

73. Vakili, R.; Xu, S.; Al-Janabi, N.; Gorgojo, P.; Holmes, S.M.; Fan, X. Microwave-assisted synthesis of zirconium-based metal organic frameworks (MOFs): Optimization and gas adsorption. Microporous Mesoporous Mater. 2018, 260, 45-53. [CrossRef]

74. Nayak, S.N.; Bhasin, C.P.; Nayak, M.G. A review on microwave-assisted transesterification processes using various catalytic and non-catalytic systems. Renew. Energy 2019, 143, 1366-1387. [CrossRef]

75. Gude, V.G.; Patil, P.D.; Deng, S.; Khandan, N. Microwave-Enhanced Methods for Biodiesel Production and Other Environmental Applications. In Green Chemistry for Environmental Remediation; Wiley Interscience: New York, NY, USA, 2011; pp. $209-249$. [CrossRef]

76. Perreux, L.; Loupy, A. A tentative rationalization of microwave effects in organic synthesis according to the reaction medium, and mechanistic considerations. Tetrahedron 2001, 57, 9199-9223. [CrossRef]

77. Tierney, J.; Lidström, P. Microwave Assisted Organic Synthesis; John Wiley \& Sons: Hoboken, NJ, USA, 2009.

78. Patil, P.D.; Deng, S. Transesterification of camelina sativa oil using heterogeneous metal oxide catalysts. Energy Fuels 2009, 23, 4619-4624. [CrossRef]

79. Dhawane, S.H.; Kumar, T.; Halder, G. Biodiesel synthesis from Hevea brasiliensis oil employing carbon supported heterogeneous catalyst: Optimization by Taguchi method. Renew. Energy 2016, 89, 506-514. [CrossRef]

80. Shuit, S.H.; Lee, K.T.; Kamaruddin, A.H.; Yusup, S. Reactive extraction of Jatropha curcas L. seed for production of biodiesel: Process optimization study. Environ. Sci. Technol. 2010, 44, 4361-4367. [CrossRef] [PubMed] 